Bull. Mater. Sci., Vol. 20, No. 6, September 1997, pp. 787-803. (C) Printed in India.

\title{
Ordering in Ni-Mo alloys-First-principles calculations versus experimental observations
}

\author{
S BANERJEE, A ARYA* and G P DAS ${ }^{\dagger}$ \\ Materials Science Division, ${ }^{\dagger}$ Technical Physics and Prototype Engineering Division, Bhabha \\ Atomic Research Centre, Bombay 400 085, India
}

\begin{abstract}
An extensive amount of experimental work has been reported in the literature on the ordering behaviour of Ni-Mo alloys containing 8-33 at $\%$ of Mo, which exhibit both short-range and long-range ordering phenomena and a competition among several fcc-based long-range ordered structures. We have used local-density-based tight binding linear muffin-tin orbital (TB-LMTO) method in conjunction with 'augmented space recursion + orbital peeling' $(A S R+O P)$ for the determination of ground state energies of these superstructures in terms of effective pair interactions up to the fourth nearest neighbour pairs. The ordering behaviour of the four competing fcc-based superstructures has been studied using the mean-field-based 'static concentration wave' (SCW) model in terms of the free energy-order parameter plots (Landau plots) and the free energy-composition plots. The instability domains with respect to concentration fluctuations, both short wavelength (ordering) and long wavelength (clustering) have been identified from these calculations. This information has been used to predict the sequence of transformation events in the Ni-Mo alloys undergoing ordering and/or clustering and the results are compared with those obtained experimentally.
\end{abstract}

Keywords. Ni--Mo alloys; first-principles calculations; phase stability; order-disorder transformations; configurational thermodynamics.

\section{Introduction}

The Ni-rich side of the Ni-Mo system has three equilibrium ordered intermetallic phases viz. $\beta-\mathrm{Ni}_{4} \mathrm{Mo}\left(\mathrm{D} 1_{a}\right), \gamma-\mathrm{Ni}_{3} \mathrm{Mo}\left(\mathrm{DO}_{a}\right)$ and $\delta$-NiMo $\left(P 2,2_{1} 2_{1}\right)$. Some unique features of the ordering transformation in this system have attracted the attention of several research groups. An extensive amount of research work (Westbrook 1967; Kear et al 1970; Warlimont 1974; Brooks et al 1984) has been dedicated to understand the behaviour of phase transformations that occur in this system, involving both short-range ordering (SRO) and long-range ordering (LRO) processes (Guthrie and Stansbury 1961; Casselton and Hume-Rothery 1964; Heijwegen and Rieck 1973; Banerjee et al 1984, 1989). This alloy system is particularly interesting from the objective of studying ordering reactions in which several fcc-based superstructures compete with each other. Further, one also observes, in this system, a competition between several first-order and a second-order ordering transformations which generate spectacular microstructures consisting of a juxtaposition of several coherent ordered structures. The relative stabilities of these competing structures, computed from the present theoretical approach can be compared with the extensive experimental data available on this system.

\footnotetext{
*Author for correspondence
} 


\section{Ordering in Ni-Mo alloys: An overview}

The Ni-Mo phase diagram (Hansen and Anderko 1958; Shunk 1969; Brooks et al 1984) shows several equilibrium-ordered intermetallic phases viz. $\beta$-phase $\left(\mathrm{Ni}_{4} \mathrm{Mo}\right)$, $\gamma$-phase $\left(\mathrm{Ni}_{3} \mathrm{Mo}\right), \delta$-phase $(\mathrm{NiMo})$, and metastable phases such as $\mathrm{Ni}_{2} \mathrm{Mo}\left(\mathrm{Pt}_{2} \mathrm{Mo}\right)$, $\mathrm{Ni}_{3} \mathrm{Mo}\left(\mathrm{DO}_{22}\right)$ and $\mathrm{Ni}_{2} \mathrm{Mo}_{2}($ I4 $/$ lamd $)$. The coherent LRO structures, which are encountered in this alloy system, can be described in terms of periodic stacking of all $\mathrm{Ni}$ and all Mo layers on $\{420\}$ planes as shown in figure 1 . These members of the (420) series are generally designated as $N_{4} M, N_{3} M, N_{2} M$ and $N_{2} M_{2}$ where $\mathrm{N}$ and $\mathrm{M}$, respectively, represent the majority and the minority atoms. There have been a number of studies on the ordering of alloys based on $\mathrm{Ni}_{4} \mathrm{Mo}$ and $\mathrm{Ni}_{3} \mathrm{Mo}$. It has been well established that these alloys, on quenching from the single phase fcc region, produce a short-range ordered (SRO) state characterized by diffraction intensity at $\left\{1 \frac{1}{2} 0\right\}_{\text {fec }}$ positions (see figure 2) and a complete extinction of intensity at $\{210\}_{\text {fec }}$ positions of the reciprocal space (Das et al 1973; Das and Thomas 1974; Ridder et al 1976; Yamamoto et al 1978; Martin and Williams 1984). Such an SRO structure has been observed over a range of compositions (8-33 at\% Mo) in $\mathrm{Ni}-\mathrm{Mo}$ alloys and in several other alloys like $\mathrm{Au}_{4} \mathrm{~V}, \mathrm{Au}_{4} \mathrm{Fe}, \mathrm{Au}_{4} \mathrm{Mn}, \mathrm{Au}_{3} \mathrm{Cr}$ (Das et al 1973; Tendeloo et al 1985).

The development of the LRO structures from the SRO state in the $\left\{1 \frac{1}{2} 0\right\}$ family of alloys has often been found to occur through a variety of intermediate stages (Ridder et al 1976; Tendeloo 1976; Banerjee et al 1984). In $\mathrm{Ni}_{4} \mathrm{Mo}$-based alloys, the initial stages of the SRO to LRO transition have been found to proceed in a
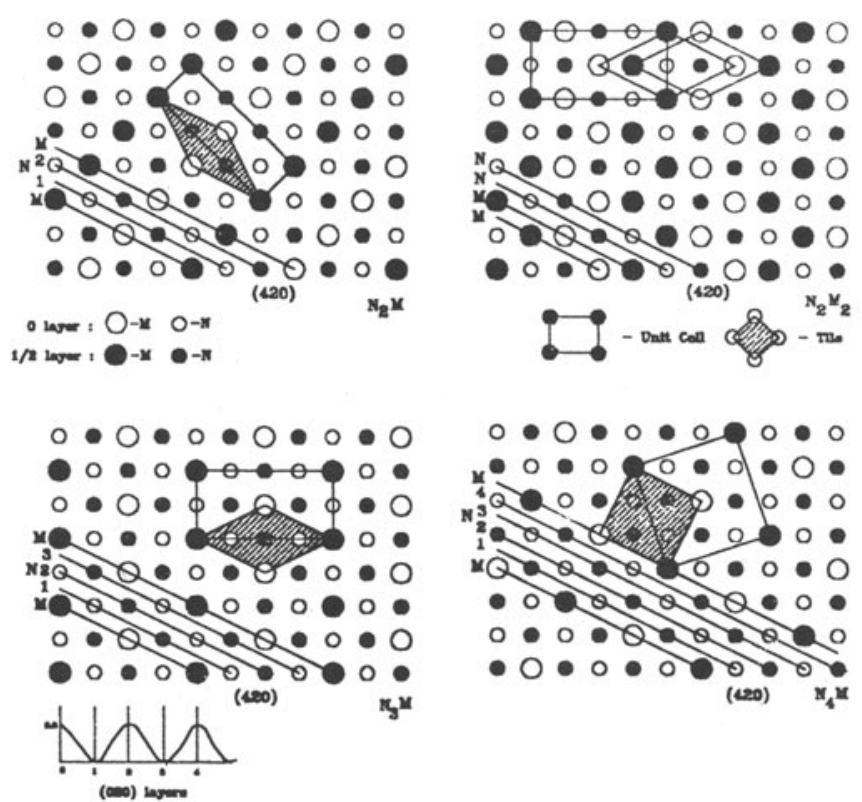

Figure 1. The description of four fcc-based superstructures in terms of stacking of (420) planes in the [001] projections. The sequences of $\mathrm{Ni}(\mathrm{N})$ and $\mathrm{Mo}(\mathrm{M})$ layers of (420) planes and subunit cell clusters (tiles) are also shown (Kulkarni and Banerjee 1988). 
continuous manner in a certain temperature range as evidenced by a continuous transfer of intensity from $\left\{1 \frac{1}{2} 0\right\}$ to $\frac{1}{5}\{420\}$ positions. At higher temperatures, however, the transition occurs by nucleation and growth of $\mathrm{D} 1_{a}: \mathrm{Ni}_{4} \mathrm{Mo}$ particles. In $\mathrm{Ni}_{3} \mathrm{Mo}$-based alloys, the SRO to LRO transition has been observed to be more complex in that the SRO state initially decomposes into a phase mixture of two

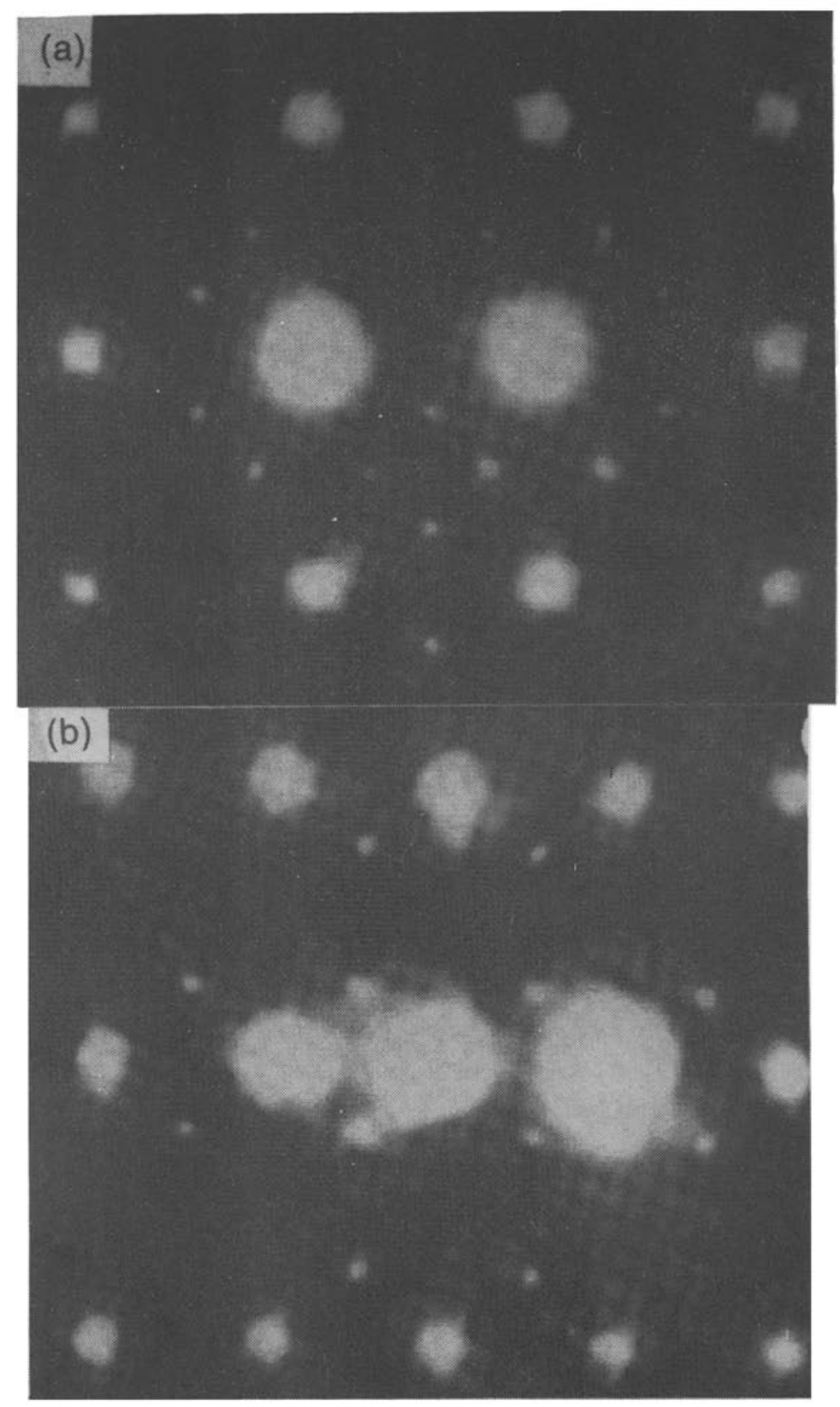

Figure 2. Diffraction pattern (a) [001] zone and (b) [112] zone axis, showing intensity maxima at $\left\langle 1 \frac{1}{2} 0\right\rangle^{*}$ positions characteristic of the SRO structure in Ni-Mo alloys. 
coherent ordered phases: (i) the metastable $\mathrm{Ni}_{2} \mathrm{Mo}\left(\mathrm{Pt}_{2} \mathrm{Mo}\right.$ type, $I m m m$ ), and (ii) the $\mathrm{Ni}_{4} \mathrm{Mo}\left(\mathrm{D} l_{a}, B 2 / m\right)$. The stoichiometric $\mathrm{DO}_{22}$ structure has been found to form only under some special circumstances e.g. in ternary alloys where Mo is partially substituted by $\mathrm{Al}$ or Ta or in alloys aged at temperatures below $900 \mathrm{~K}$ for a long time.

The general tendency of the $\mathrm{Ni}_{3} \mathrm{Mo}$ alloy and some of its ternary derivatives to decompose into a mixture of the $\mathrm{Ni}_{2} \mathrm{Mo}$ and the $\mathrm{Ni}_{4} \mathrm{Mo}$ phases, avoiding the $\mathrm{DO}_{22}$ structure which satisfies the stoichiometry, has been rationalized in terms of nearly equal stabilities associated with the competing structures (Das and Thomas 1974). The calculation of formation energies, used for such comparisons, are, however, based on arbitrary values of interaction energies for the first, the second and the third nearest neighbour pairs. The present work attempts to draw the comparison on the basis of more rigorous first-principles calculations.

Let us consider an alloy composition $\mathrm{Ni}-25$ at\% $\mathrm{Mo}$, since no off-stoichiometric structure can be stable at $0 \mathrm{~K}$ due to the entropy consideration, the $\mathrm{N}_{2} \mathrm{M}_{2}$ structure for this composition cannot be stable. In such a situation, the alloy is expected to undergo a secondary ordering giving rise to a stoichiometric ordered structure or separate into a phase mixture of an ordered structure and a pure component. Kulkarni and Banerjee (1988) considered three different cases of secondary ordering, involving three different fcc special point concentration waves in the off-stoichiometric $\mathrm{N}_{2} \mathrm{M}_{2}$ structure generated by $\left\langle 1 \frac{1}{2} 0\right\rangle^{*}$ spinodal ordering: (i) generation of long wave-length concentration waves with $\mathbf{k}$-vectors close to $\langle 000\rangle^{*}$ in an off-stoichiometric partially $\left\langle 1 \frac{1}{2} 0\right\rangle^{*}$ ordered alloy results in a phase separation of the alloy into a disordered solid solution and a nearly stoichiometric ordered $\mathrm{N}_{2} \mathrm{M}_{2}$ phase, (ii) introduction of a $\langle 100\rangle^{*}$ concentration wave in a $\left\langle 1 \frac{1}{2} 0\right\rangle^{*}$ ordered alloy of $\mathrm{Ni}_{3} \mathrm{Mo}$ composition, gives rise to the $\mathrm{DO}_{22}$ structure (secondary ordering), and (iii) secondary $\left\langle 1 \frac{1}{2} 0\right\rangle^{*}$ ordering in a direction perpendicular to the original ordering $\mathbf{k}$-vector (another variant of $\left\langle 1 \frac{1}{2} 0\right\rangle^{*}$ ) results in the formation of a ' $N_{3} M^{\prime}$ 'structure which would contain an ordered arrangement of $\mathrm{D} 1_{a}$ and $\mathrm{Pt}_{2} \mathrm{Mo}$ subunit cell clusters, each being made up of one 'molecule' of square shaped $\mathrm{Ni}_{4} \mathrm{Mo}$ or diamond shaped $\mathrm{Ni}_{2} \mathrm{Mo}$ respectively (see figure 3 ).

The ground state analysis, reported by Kulkarni and Banerjee (1988), pointed to the fact that in an $\left\{1 \frac{1}{2} 0\right\}$ alloy of $\mathrm{Ni}_{3} \mathrm{Mo}$ stoichiometry, the superimposition of either (i) $a\left\langle 1 \frac{1}{2} 0\right\rangle^{*}$ and $a\langle 100\rangle^{*}$ concentration waves or (ii) two mutually perpendicular $\left\langle 1 \frac{1}{2} 0\right\rangle^{*}$ concentration waves always results in a structure of lower internal energy than the off-stoichiometric structure generated by a single $\left\langle 1 \frac{1}{2} 0\right\rangle^{*}$ concentration wave. Moreover, the internal energy of an equimolar mixture of the $\mathrm{Ni}_{2} \mathrm{Mo}$ and the $\mathrm{Ni}_{4} \mathrm{Mo}$ phases is same as that of the $\mathrm{Ni}_{3} \mathrm{Mo}$.

The occurrence of the $\left\langle 1 \frac{1}{2} 0\right\rangle^{*}$ instability and the stability of the structures belonging to this family can be associated with the 'like' third neighbour minority atom (Mo-Mo) interactions (negative value of $J_{2}^{(3)}$ ). On the other hand, the $\langle 100\rangle^{*}$ instability and the structures belonging to this family $\left(\mathrm{L1}_{2}, \mathrm{L1}_{0}\right)$ would be characterized by the 'unlike' third neighbour interactions or positive value of $J_{2}^{(3)}$.

It is interesting to note that subunit cell tiles corresponding to the $N_{4} M, N_{3} M$ and $\mathrm{N}_{2} \mathrm{M}$ structures, as indicated in figure 4 , can decorate an fcc lattice with perfect space filling. This is illustrated in the figure in which superlattice tiles of all the three types are present in a quasi-periodic array decorating the underlying fcc lattice. Such structure can be generated by projecting 4-D simple cubic on a 2-D subspace. The details of the projection formalism has been reported by Kulkarni 


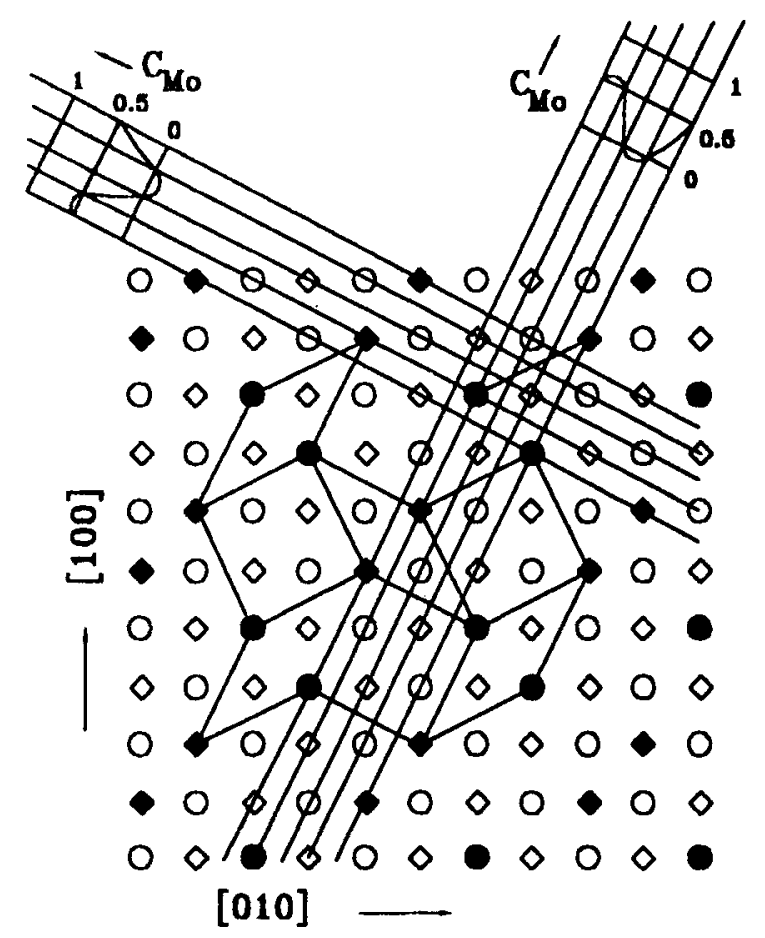

Figure 3. [001] projection showing atomic arrangement in the ' $N_{3} M$ structure' generated by the superimposition of two perpendicular $\left\langle 1 \frac{1}{2} 0\right\rangle^{*}$ concentration waves. This structure comprises an arrangement of two variants, each of subunit cell clusters corresponding to the $\mathrm{N}_{4} \mathrm{M}$ (square-shaped) and the $\mathrm{N}_{2} \mathrm{M}$ (diamond-shaped) structures (Kulkarni and Banerjee 1988).

et al (1993). Their illustration brings out the fact that the superlattice tiles, corresponding to the competing superstructures, can form a quasi-periodic superlattice structure on a periodic lattice (figure 4). Experimental verification of such a structure has still not been possible, though co-existence of several sub-unit cell clusters during the evolutionary stages of ordering in $\mathrm{Ni}-\mathrm{Mo}$ system has been revealed, both in diffuse intensity distribution in the diffraction pattern and in HREM images.

\section{The first-principles approach}

The approach to the first-principles configurational thermodynamic studies of alloys can be schematically illustrated in a flow chart (figure 5). A brief account of each method is given as follows:

\subsection{Determination of total energies $\left(E_{T}^{\Phi}(V)\right)$}

Determination of total energies $\left(E_{T}^{\Phi}(V)\right)$ of all the superstructures $(\Phi)$ as a function of volume $(V)$, of a given alloy system, is given by using some local density-based electronic structure method. We have employed the state-of-art tight-binding linear muffin-tin orbital (TB-LMTO) method (Andersen et al 1985, 1987) in the simplifying 
atomic sphere approximation (ASA). In the ASA, the matrix elements of the one electron Hamiltonian in a nearly orthogonal LMTO representation $(\gamma)$ can be written as

$$
H_{\mathbf{R L}, \mathbf{R}^{\prime} \mathbf{L}^{\prime}}=C_{\mathbf{R L}^{\prime}} \delta_{\mathrm{RR}^{\prime}} \delta_{\mathbf{L L}^{\prime}}+\Delta_{\mathbf{R L}}^{\mathrm{l} / 2}\left[S^{o}\left(1-\gamma S^{o}\right)\right]_{\mathbf{R L}, \mathbf{R}^{\prime} \mathbf{L}^{\prime}} \Delta_{\mathbf{R}^{\circ}}^{\mathrm{l} / 2},
$$

where $\mathbf{R}$ refers to the site index and $\mathbf{L}(\equiv l m)$ refers to the orbital-angular momentum index. $S$ is the canonical structure constant matrix, which depends on the relative positions of the sites and is independent of the type of atoms occupying the sites. The properties of the atoms are completely described by the potential parameter matrices $C, \Delta$, and $\gamma$ which are diagonal in the indices $\mathbf{R}$ and $\mathbf{L}$. The Löwdinorthogonalized Hamiltonian in ASA, $\mathbf{O}^{-1 / 2} \mathbf{H O}^{-1 / 2}$, can be obtained from the knowledge of an additional potential parameter ' $p$ ' which determines the overlap matrix ' $\mathbf{O}$ ':

$$
\mathbf{O}=\mathbf{I}+\mathbf{h p h}, \quad \text { with } \quad \mathbf{h}=\mathbf{H}-E_{\boldsymbol{v}} \text {, }
$$

where $I$ is a unit matrix and $E_{v}$ the reference energy of the linear method. The self-consistent potential parameters are derived from the solution of radial Schrödinger equation at the sphere boundary and its energy derivatives at the reference energy $E_{v}$. The short-ranged Hamiltonian matrix elements in TB-representation $(\alpha)$ can be

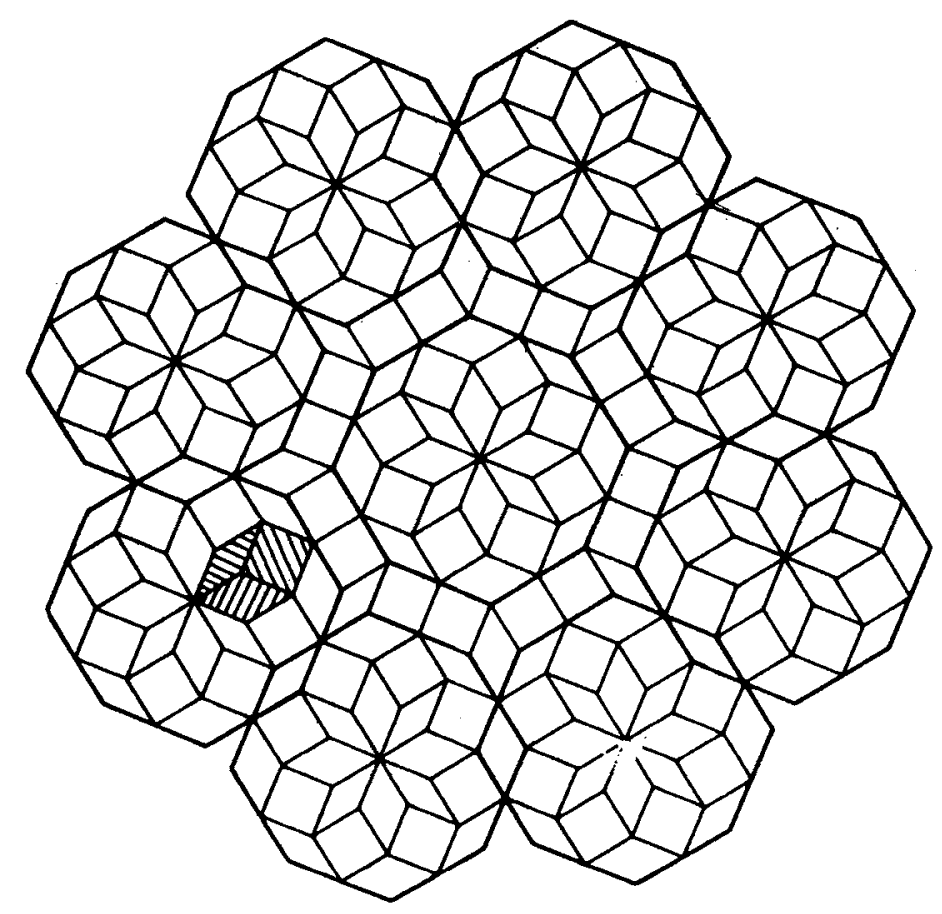

Figure 4. A quasi-periodic superlattice structure showing the tiles corresponding to $\mathrm{N}_{4} \mathrm{M}$ (square shaped), $\mathrm{N}_{2} \mathrm{M}$ (tilted rhombus) and $\mathrm{N}_{3} \mathrm{M}\left(\mathrm{DO}_{22}\right.$, rhombus) which decorate an underlying simple cubic lattice filling the space completely and maintaining the coherency. This structure is obtained by taking a projection of the 4-D single cubic structure on a 2-D subspace. 
obtained by using the prescription of Andersen and Jepsen (Andersen et al 1985). The Löwdin-orthogonalized Hamiltonian can be expressed in terms of TB Hamiltonian $\left(\mathbf{H}^{\alpha}\right)$ as

$$
\mathbf{H}=\mathbf{H}^{\alpha}-\mathbf{h}^{\alpha} \mathbf{0}^{\alpha} \mathbf{h}^{\alpha}+\ldots
$$

From these ASA total energy $\left(E_{T}^{\Phi}\right)$ values obtained by solving the Schrödinger equation, one can calculate the equilibrium volume $\left(V_{0}^{\Phi}\right)$ (by minimizing $E_{T}^{\Phi}(V)$ with respect to global volume), the bulk modulus $\left[B^{\Phi} \equiv(1 / V)\left(\partial^{2} E_{T}^{\Phi} / \partial V^{2}\right)\right]$, the cohesive energy $\left[E_{\mathrm{coh}}^{\Phi}=E_{T}^{\Phi}-\Sigma_{i} E_{i}^{\text {atomic }}\right]$ and the energy of formation $\left[E_{\text {form }}^{\Phi}=E_{\text {coh }}^{\Phi}-\Sigma_{i} E_{i}^{o}\right]$, where $E_{\mathrm{A}(\mathrm{B})}^{\text {atomic }}$ is the atomic energy of component $\mathrm{A}(\mathrm{B})$ and $E_{\mathrm{A}(\mathrm{B})}^{o}$ is the cohesive energy of the pure $\mathrm{A}(\mathrm{B})$ having the same parent lattice as that of $\Phi$.

\subsection{Determination of configurationally-averaged effective pair interactions (EPIs)}

These EPIs serve as an input to the calculation of configurational energy of the system and can be calculated using the 'augmented space recursion (ASR) + orbital peeling (OP)' technique (Mookerjee 1994). The recursion method is an alternative to the band-structure method and is most useful where Bloch periodicity is absent and one is interested in the local properties of the alloy. In ASR; the recursion is performed in the augmented space (Hilbert $(\mathcal{H})+$ Configurational $(C)$ ) using tight-

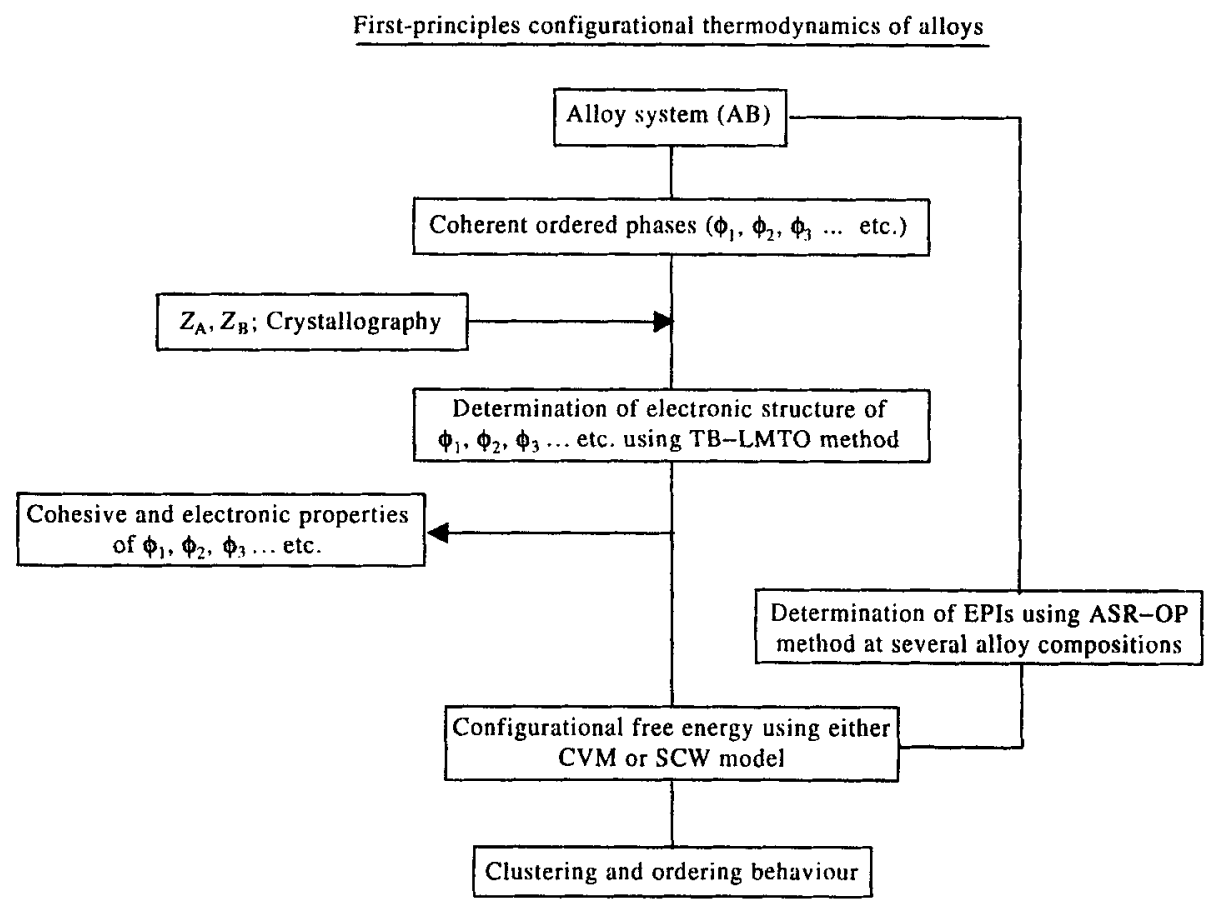

Figure 5. A flow chart illustrating the overall scheme of calculation 
binding Hamiltonian which is sparse in nature. The ASR in conjunction with orbital peeling technique is a first-principle tool for obtaining the configurationally-averaged EPIs up to an arbitrary coordination shell.

\subsection{Determination of configurational free energy}

The static concentration wave (SCW) model: In the SCW model (Khachaturyan 1973, 1983), the occupation probability $(\mathcal{N}(\mathbf{R}))$ at a given site $(\mathbf{R})$ is Fourier expanded as a sum of static concentration waves having wave vector ' $\mathbf{k}$ ' and amplitude $Q(\mathbf{k})$ as

$$
\mathcal{N}(\mathbf{R})=c+\sum_{k} Q(\mathbf{k}) \exp (i \mathbf{k} \cdot \mathbf{r})
$$

where $c$ is the bulk concentration and the amplitude $Q(\mathbf{k})$ is proportional to the order parameter $(\eta)$. The internal energy of the system as a function of amplitude of the $\mathrm{CW}$ is given as

$$
E=\frac{1}{2} N \sum_{\mathbf{k}_{i}} J\left(\mathbf{k}_{i}\right) Q^{*}\left(\mathbf{k}_{i}\right) Q\left(\mathbf{k}_{i}\right)
$$

where $N$ is the total number of lattice sites and $J(\mathbf{k}) \mathrm{s}$ are the Fourier transform of the EPIs. The configurational entropy, in the mean-field approximation, is given by

$$
S=-k_{\mathrm{B}} \sum_{i=1}^{N}\left[\mathcal{N}\left(\mathbf{R}_{i}\right) \ln \mathcal{N}\left(\mathbf{R}_{i}\right)+\left(1-\mathcal{N}\left(\mathbf{R}_{i}\right)\right) \ln \left(1-\mathcal{N}\left(\mathbf{R}_{i}\right)\right)\right],
$$

where $k_{\mathrm{B}}$ is the Boltzmann constant. The free energy $(F=E-T S)$ is, thus, obtained as a function of temperature $(T)$, composition $(c)$ and order parameter $(\eta)$.

\subsection{Ordering and clustering behaviour}

The precipitation of an ordered phase $(\beta)$ from a disordered solid solution $(\alpha)$ involves ordering and/or clustering processes occurring either sequentially or simultaneously. The actual thermodynamic behaviour is generally governed by the presence of instabilities with respect to clustering and ordering in the $\alpha$-phase. Depending upon the sign and magnitude of EPIs, the $\alpha$-phase can be stable/metastable/unstable with respect to ordering or clustering. The sequence of transformations leading to the formation of $\beta$-phase is predicted in terms of relative positions of the instability lines. These instability lines are (i) ordering spinndal line $\left(T_{i}^{-}\right)$of the disordered phase, (ii) chemical spinodal line $\left(T_{\mathrm{s}}\right)$ of the disordered phase, (iii) the $T_{i}^{+}$line above which ordered phase becomes unstable with respect to congruent spontaneous disordering and (iv) clustering spinodal line $\left(T_{c s}\right)$ of the ordered phase.

\section{Results and discussion}

\subsection{The ground state stability}

For our theoretical investigation of ground state phase stability, we have considered 
12 fcc-based coherent structures (see table 1) in the entire concentration range, apart from the two equilibrium structures viz. Mo (bcc) and the $\mathrm{Ni}_{3} \mathrm{Mo}\left(\mathrm{DO}_{a}\right.$ ) structure (Arya et al 1997a). $\mathrm{DO}_{a}: \mathrm{Ni}_{3} \mathrm{Mo}$ is the only hcp-based structure included here because in $75: 25$ composition, this is the stable structure closely competing with the related fcc-based $\mathrm{DO}_{22}: \mathrm{Ni}_{3} \mathrm{Mo}$ structure.

The average Wigner Seitz (WS) radii of various $\mathrm{Ni}-\mathrm{Mo}$ intermetallics are found to be inversely proportional to their e/a ratio (see table 1). For each of these structures, we have performed self-consistent calculations for global minimization of the total ground state energy with respect to the cell volume. Using different atomic sphere radii for $\mathrm{Ni}$ and $\mathrm{Mo}$, as given by the compressibility criterion (Andersen et al 1985), one gets more or less charge-neutral spheres. These values of $s_{\mathrm{Ni}}$ and $s_{\mathrm{Mo}}$ for each superstructure are given in table 1. The resulting equilibrium $s_{\mathrm{av}}$ values are found to be slightly higher (systematically) than the corresponding Vegard's law values, although the numbers follow the same increasing trend with the increase of Mo-concentration. This small positive deviation from the Vegard's

Table 1. Table of calculated ground state properties of various superstructures of Ni-Mo alloy system (Arya et al 1997a).

\begin{tabular}{|c|c|c|c|c|c|}
\hline $\begin{array}{l}\text { Structures } \\
\text { fcc-based }\end{array}$ & $e / a$ & $\begin{array}{c}S_{\mathrm{itv}} \\
\left(S_{\mathrm{Nj}}, S_{\mathrm{Mu}}\right) \\
\text { (a.u.) }\end{array}$ & $\begin{array}{l}\text { Bulk mod. } \\
\text { (GPa) }\end{array}$ & $\begin{array}{c}E_{\mathrm{colh}} \\
(\mathrm{kJ} / \mathrm{mol})\end{array}$ & $\underset{(\mathrm{kJ} / \mathrm{mol})}{E_{\mathrm{lorm}}}$ \\
\hline $\mathrm{Ni}(\mathrm{fcc})$ & $10 \cdot 0$ & 2.500 & $256 \cdot 268$ & -791.563 & 0.0 \\
\hline $\mathrm{Ni}_{4} \mathrm{Mo}$ & $9 \cdot 2$ & $\begin{array}{c}2.636 \\
(2.543,2.952)\end{array}$ & $298 \cdot 232$ & -878.942 & $-25 \cdot 322$ \\
\hline $\mathrm{Ni}_{3} \mathrm{Mo}\left(\mathrm{DO}_{Q}\right)$ & 9.0 & $\begin{array}{c}2.656 \\
(2.542,2.951)\end{array}$ & 299.484 & $-894 \cdot 154$ & -25.021 \\
\hline $\mathrm{Ni}_{3} \mathrm{Mo}\left(\mathrm{LL}_{2}\right)$ & 9.0 & $\begin{array}{c}2.662 \\
(2.548,2.957)\end{array}$ & 248.609 & -876.189 & -7.055 \\
\hline $\mathrm{Ni}_{3} \mathrm{Mo}\left(\mathrm{DO}_{22}\right)$ & $9 \cdot 0$ & $\begin{array}{c}2.653 \\
(2.539,2.947)\end{array}$ & 258.678 & -893.540 & $-24 \cdot 406$ \\
\hline $\mathrm{Ni}_{2} \mathrm{Mo}$ & 8.67 & $\begin{array}{c}2 \cdot 691 \\
(2 \cdot 540,2 \cdot 948)\end{array}$ & 258.011 & -927.099 & $-32 \cdot 109$ \\
\hline NiMo $\left(\mathrm{LI}_{0}\right)$ & 8.0 & $\begin{array}{c}2.757 \\
(2.538,2.946)\end{array}$ & 91.586 & -971.583 & $-24 \cdot 879$ \\
\hline $\mathrm{Ni}_{2} \mathrm{Mo}_{2}$ & 8.0 & $\begin{array}{c}2.788 \\
(2.568,2.978)\end{array}$ & 208.916 & -961.409 & -14.705 \\
\hline NiMo $\left(\mathrm{L} 1_{1}\right)$ & 8.0 & $\begin{array}{c}2.790 \\
(2.570,2.980)\end{array}$ & 334.423 & $-969 \cdot 644$ & -22.940 \\
\hline $\mathrm{NiMo}_{2}$ & 7.33 & $\begin{array}{c}2.842 \\
(2.555,2.964)\end{array}$ & 242.533 & $-1008 \cdot 604$ & -10.186 \\
\hline $\mathrm{NiMo}_{3}\left(\mathrm{Ll}_{2}\right)$ & 7.0 & $\begin{array}{c}2.856 \\
(2.539,2.947)\end{array}$ & $225 \cdot 490$ & -1050.957 & -26.682 \\
\hline $\mathrm{NiMo}_{3}\left(\mathrm{DO}_{22}\right)$ & 7.0 & $\begin{array}{c}2.862 \\
(2.545,2.953)\end{array}$ & $255 \cdot 108$ & $-1042 \cdot 724$ & -18.450 \\
\hline Mo (fcc) & 6.0 & 2.959 & 279.411 & -1101.845 & 0.0 \\
\hline Mo (bcc) & 6.0 & 2.959 & 283.902 & $-1140 \cdot 265$ & 0.0 \\
\hline
\end{tabular}


law indicates the manifestation of solid solution effects in Ni-Mo alloys. All the calculated cohesive properties have been summarized in table 1 . The bulk moduli, obtained from the second derivative of the total energy with respect to volume, remain more or less same, lying within a narrow band for all the structures; NiMo $\left(\mathrm{L1}_{0}\right)$ is the only exception for which it abruptly drops by a factor of 3 .

From the ASA total energy, we have calculated the cohesive energy $\left(E_{\mathrm{coh}}\right)$ for each superstructure by subtracting the weighted sum of total atomic energies which are $-3037.080 \mathrm{Ry}$ for $\mathrm{Ni}$ and $-8090.228 \mathrm{Ry}$ for Mo. Our calculated $E_{\text {coh }}$ values are found to increase with increasing $\mathrm{Ni}$ concentration (i.e. increasing $e / a$ ). At $x_{\mathrm{Mo}}=0.25$, the $E_{\mathrm{form}}$ for hcp-based $\mathrm{DO}_{a}$ and the fcc-based $\mathrm{DO}_{22}$ structures are found to be very close, although the former is stabler, as also confirmed by experiments (Tendeloo et al 1985; Kulkarni and Banerjee 1988). Similar argument, extended to the Mo-rich side, shows $\mathrm{NiMo}_{3}\left(\mathrm{Ll}_{2}\right)$ to be the stable structure, while $\mathrm{NiMo}_{3}\left(\mathrm{DO}_{22}\right)$ would be metastable.

\subsection{The effective pair interactions}

In view of the fact that the stabilities of ordered intermetallic phases in the Ni-Mo system can be assessed by considering up to the third or the fourth nearest-neighbour pair interactions, we have calculated the configurationally-averaged concentrationdependent EPIs $\left(J_{2}(c)\right.$ ), up to the fourth nearest neighbour pairs for the Ni-Mo alloys, using the first-principles ASR-OP technique (Arya et al 1997b). The method uses the most-localized (tight-binding) set of potential parameters in the Hamiltonian. We have used elemental TB-LMTO potential parameters, which are ideally suited for this purpose, in order to parametrize the alloy Hamiltonian. These EPIs in the $m$ th coordination sphere are defined as

$$
J_{2}^{(m)}=V_{\mathrm{AA}}^{(m)}+V_{\mathrm{BB}}^{(m)}-V_{\mathrm{AB}}^{(m)}-V_{\mathrm{BA}}^{(m)}
$$

which gives the interchange energy associated with two sites, embedded in an otherwise average medium, being occupied by either A or B atom. Table 2 gives the calculated values of the EPIs up to the fourth coordination shell for fcc-based $\mathrm{Ni}-\mathrm{Mo}$ alloys at 20,25, 33 and 50 at\% of Mo.

\subsection{The SCW analysis}

The concentration wave description of these superstructures is obtained by Fourier transforming the concentration delta function $\mathcal{N}(\mathbf{p})$, which is equal to unity at plane $\mathbf{p}=0, N, 2 N$ etc and zero elsewhere $(\mathbf{p}=1,2, \ldots N-1)$, so that the concentration Fourier spectrum is given by

$$
\mathcal{N}(\mathbf{k})=\frac{1}{N} \sum_{\mathbf{p}=0}^{N-1} \mathcal{N}(\mathbf{p}) \exp [-2 \pi i \mathbf{k p}]=\frac{1}{N}
$$

For each structure, there are exactly $N(=2,3,4,5)$ Fourier waves of same amplitude (see figure 6). We have calculated the free energies of these superstructures, as functions of temperature, composition and order parameter (amplitude of the concentration wave). 
Table 2. The concentration dependent effective pair interactions (EPI) up to the fourth nearest neighbour pair calculated using the ASR + OP method for the Ni-Mo alloys.

\begin{tabular}{lcccc}
\hline $\begin{array}{l}\text { Composition } \\
\text { (at\% of Mo) }\end{array}$ & 20 & 25 & 33 & 50 \\
\hline$E_{F}\left(\times 10^{+2}\right)$ & -4.414 & -3.265 & -1.847 & -0.860 \\
$J_{2}^{(1)}\left(\times 10^{+2}\right)$ & 0.3692 & 0.2627 & 0.0849 & 0.0536 \\
$J_{2}^{(2)}\left(\times 10^{+3}\right)$ & 0.2125 & 0.3074 & 0.4032 & -0.3291 \\
$J_{2}^{(3)}\left(\times 10^{+3}\right)$ & -0.2647 & -0.2216 & -0.1636 & -0.1240 \\
$J_{2}^{(4)}\left(\times 10^{+4}\right)$ & 0.1164 & 0.2067 & 0.2659 & 0.2610 \\
\hline
\end{tabular}
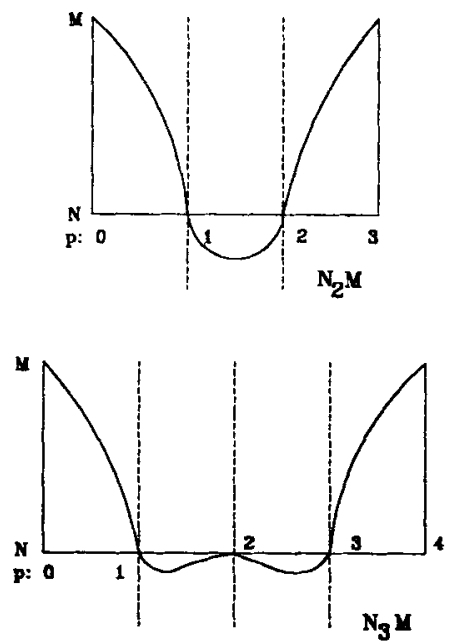
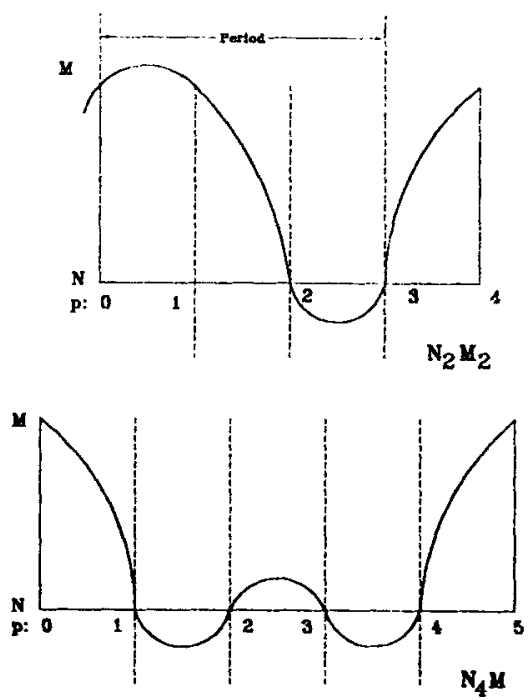

Figure 6. The concentration wave description of fcc-based coherent superstructures in terms of (420) planes containing either $\mathrm{Ni}(\mathrm{N})$ or $\mathrm{Mo}(\mathrm{M})$ atoms (de Fontaine 1975).

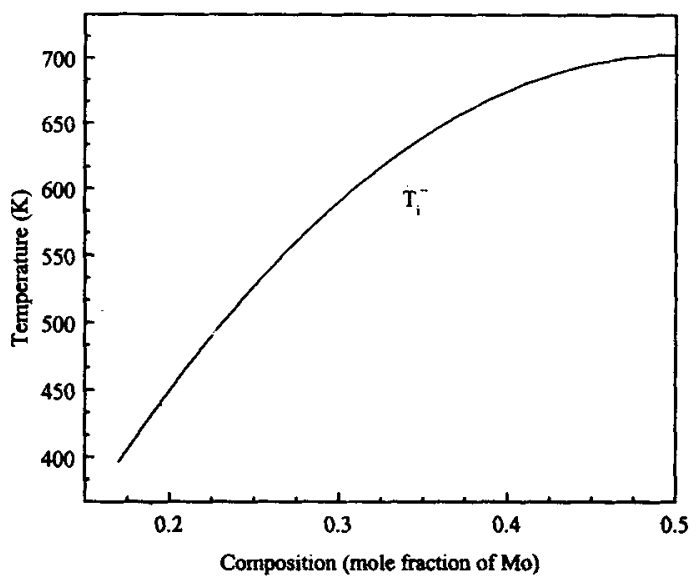

Figure 7. The temperature $T_{i}^{-}$corresponding to the onset of $\left\langle 1 \frac{1}{2} 0\right\rangle^{*}$ ordering instability plotted as a function of Mo concentration. 


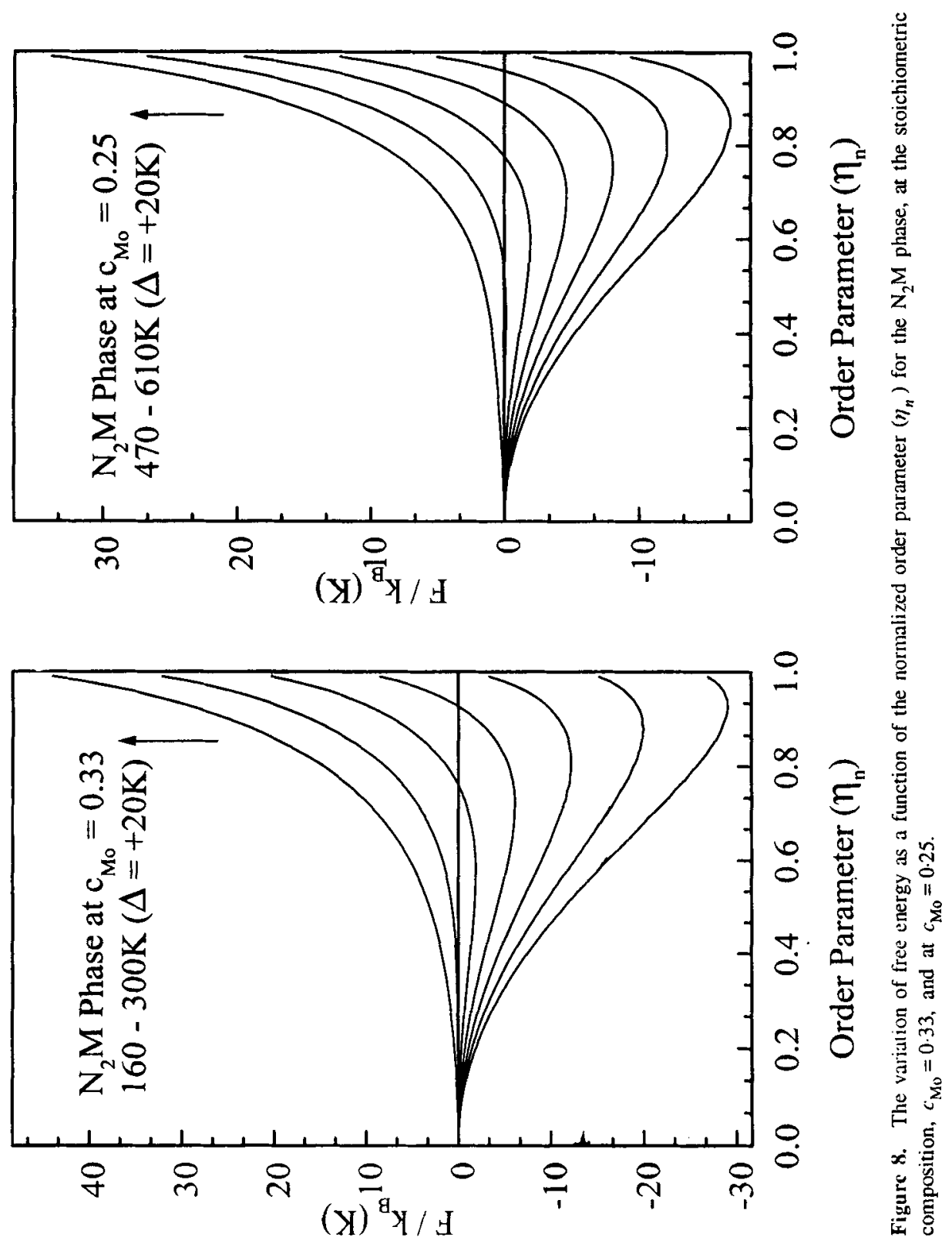




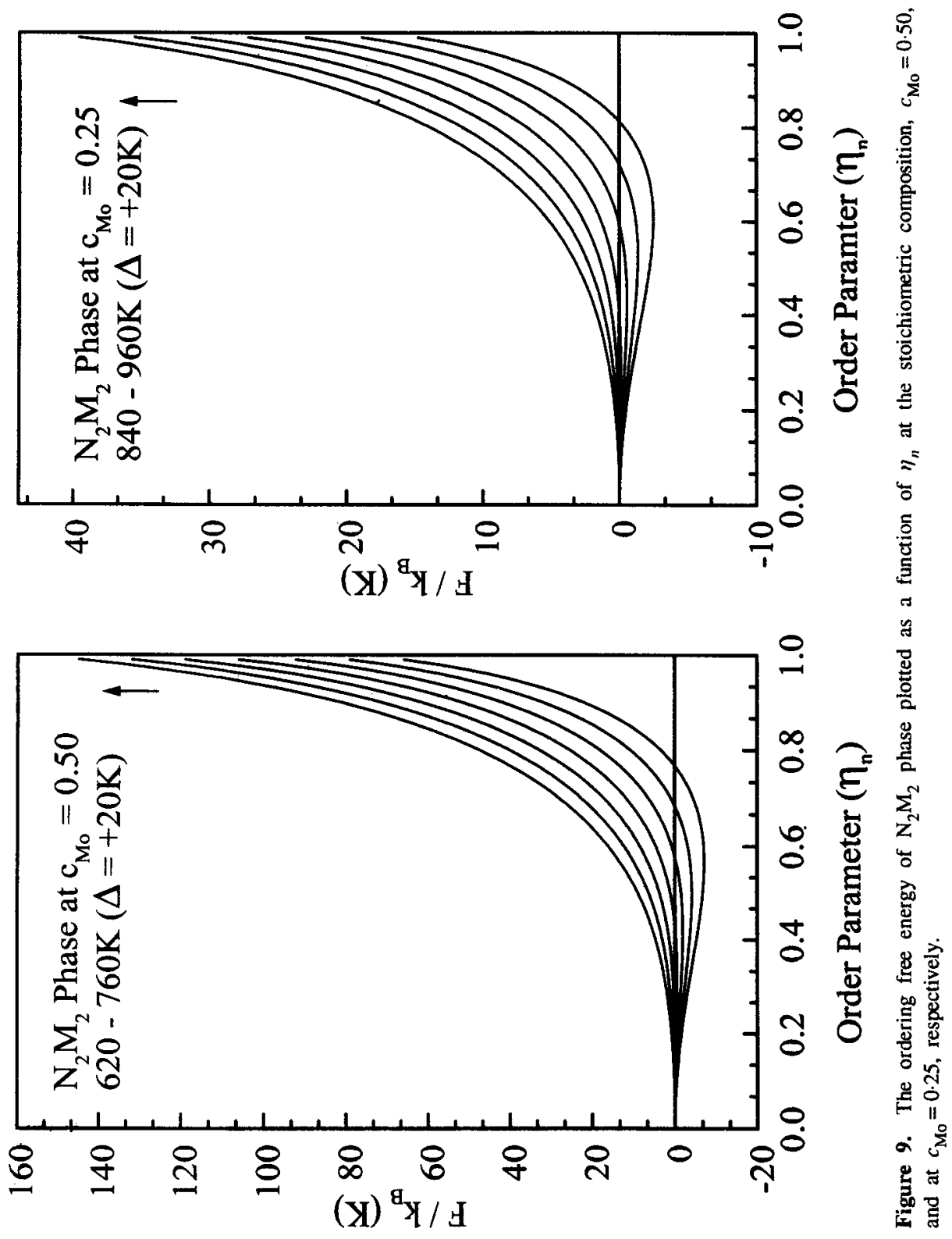


The instability temperature $\left(T_{i}^{-}\right)$, defined as the temperature corresponding to the onset of ordering instability in the solid solution, is given by (Khachaturyan 1983)

$$
T_{i}^{-}=-\frac{J(\mathbf{k})}{k_{\mathrm{B}}} c(1-c),
$$

for the ordering wave vector $k$. The locus of $\left\langle 1 \frac{1}{2} 0\right\rangle^{*}$ ordering instability for the $\mathrm{Ni}-\mathrm{Mo}$ alloys, plotted in the phase diagram (temperature $(T)$ vs composition (c) plane), is shown in figure 7 .

The variation of ordering free energy $\left(F^{\mathrm{vid}}=F^{\Phi}-F^{\text {disord }}\right)$ as a function of normalized order parameter $\left(\eta_{\mathrm{n}}\right)$ for the $\mathrm{N}_{2} \mathrm{M}$ phase, at the stoichiometric composition, $c_{\mathrm{Mo}}=0.33$, and at $c_{\mathrm{Mo}}=0.25$, is plotted in figure 8 (the so-called Landau plots) at several temperatures. These plots show a small hump in the $F^{\text {ord }}$ vs $\eta$ curve at the equilibrium temperature. This is indicative of a free energy barrier between the disordered and the ordered states characteristic of a first-order transition. At a certain degree of undercooling, an instability with respect to ordering develops as reflected by the negative curvature of the $F^{\text {ord }}$ vs $\eta$ plots at $\eta=0$.

In figure 9 , we have plotted the ordering free energy of the $\mathrm{N}_{2} \mathrm{M}_{2}$ phase as a function of $\eta_{\mathrm{n}}$ at the stoichiometric composition, $c_{\mathrm{Mo}}=0.50$, and at $c_{\mathrm{Mo}}=0.25$, respectively. The temperature range has been chosen to be around the instability temperature at a given composition. These Landau plots, generated from the

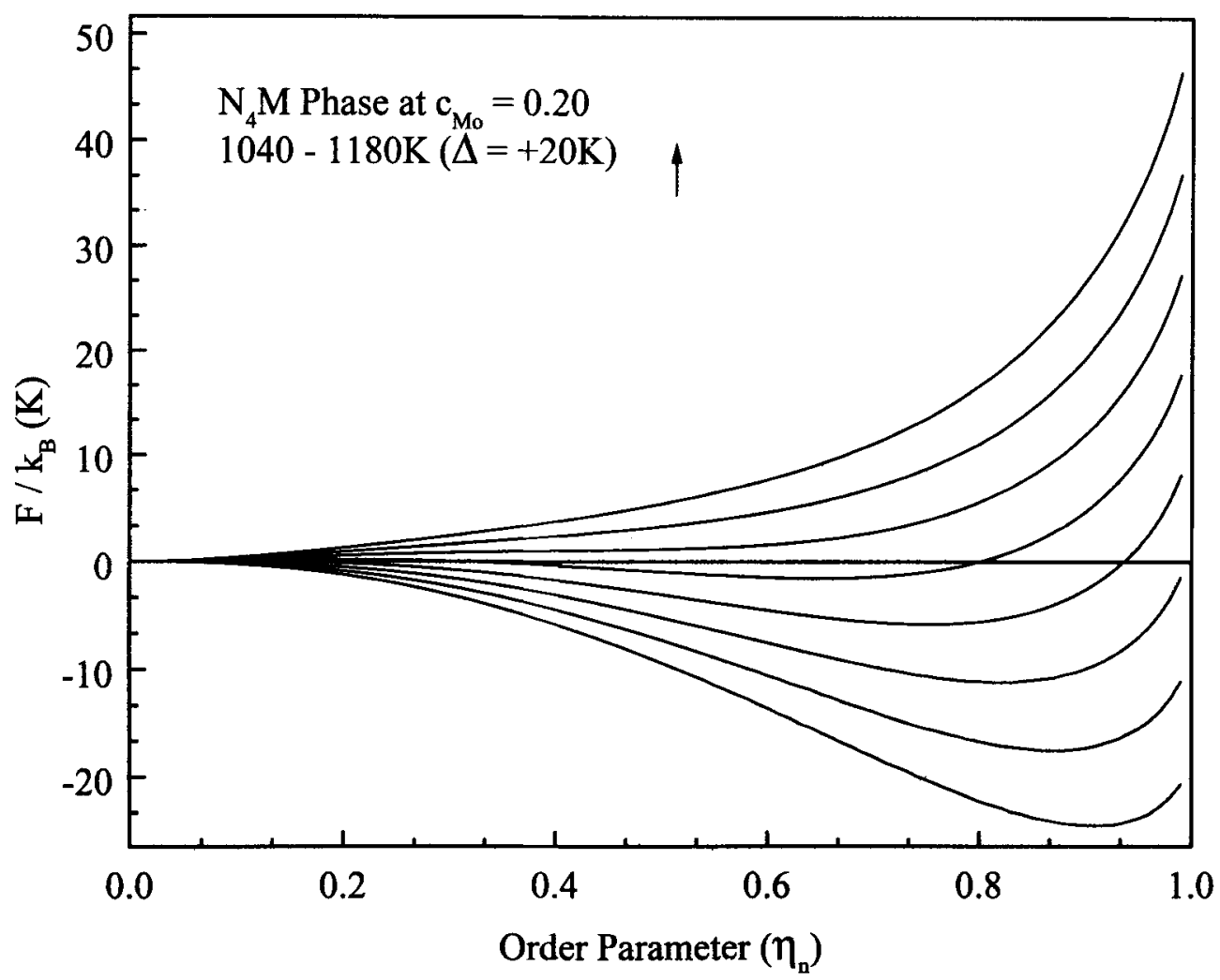

Figure 10. The variation of ordering free energy of $N_{4} M$ phase with the normalized order parameter at the stoichiometric composition $\left(c_{\mathrm{MO}}=0.2\right)$. 


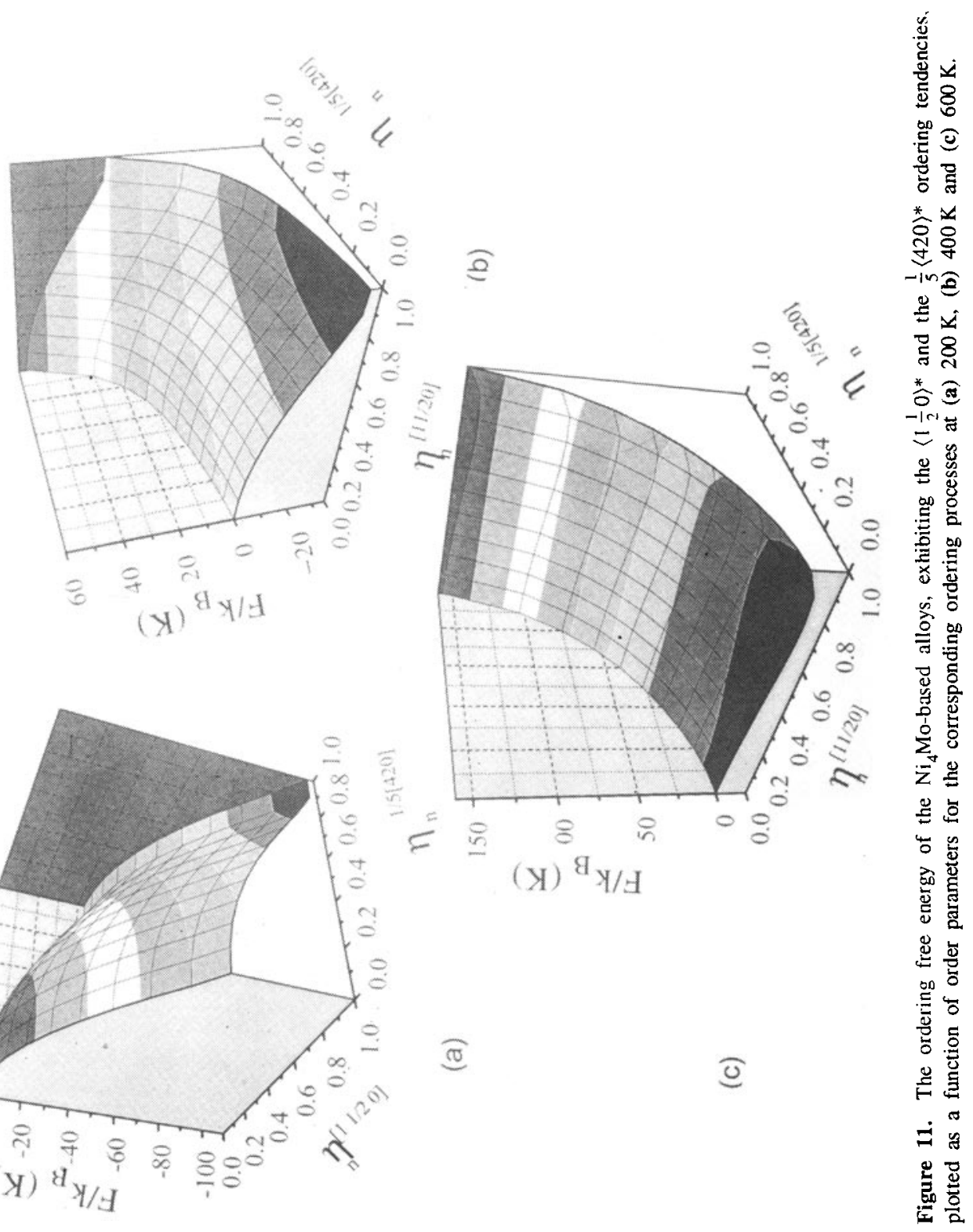


first-principles calculations, indicate that $\mathrm{N}_{2} \mathrm{M}_{2}$ ordering for a stoichiometric composition is a transformation of the second kind. The $\mathrm{N}_{2} \mathrm{M}_{2}$ instability temperatures for different alloy compositions, in the binary Ni-Mo system, essentially give the ordering spinodal line which was reported (schematically) earlier by de Fontaine (1975).

Figure 10 shows the variation of ordering free energy of the $\mathrm{D} 1_{a}$ structured $\mathrm{N}_{4} \mathrm{M}$ phase with the order parameter at the stoichiometric composition $\left(c_{\mathrm{Mu}}=0 \cdot 2\right)$. The critical temperature $\left(T_{c} \simeq 1130 \mathrm{~K}\right)$ for the $D 1_{a}$-structured $\mathrm{N}_{4} \mathrm{M}$ phases found to be in very good agreement with the experimental value $(\approx 1140 \mathrm{~K})$.

Since an alloy in this system experiences ordering tendencies corresponding to different $\mathbf{k}$-vectors, it is necessary to examine these in terms of Landau plots with respect to different $\mathbf{k}$-vectors. This point is illustrated for the $\mathrm{Ni}_{4} \mathrm{Mo}$ alloy in which the second order $\left\langle 1 \frac{1}{2} 0\right\rangle^{*}$ ordering and the first order $\frac{1}{5}\langle 420\rangle^{*}$ ordering compete. We have calculated the free energy of these alloys $F^{\mathrm{Ni}_{4} \mathrm{Mo}}\left(\eta_{n}^{\left[1 \frac{1}{2}(0)\right.}, \eta_{n}^{\left.\frac{1}{5} \mid 420\right]}\right)$ at a given temperature as

$$
F^{\mathrm{Ni}_{4} \mathrm{Mo}}\left(\eta_{n}^{\left[1 \frac{1}{2} 0\right]}, \eta_{n}^{\left.\frac{1}{\zeta \mid(42)]}\right)}=\frac{1}{2}\left[F^{\mathrm{N}_{+} \mathrm{M}}\left(\eta_{n}^{\frac{1}{5}}{ }^{[42(1)}\right)+F^{\mathrm{N}_{2} \mathrm{M}_{2}}\left(\eta_{n}^{\left[1 \frac{1}{2} 0 \mid\right.}\right)\right] \text { at } c_{\mathrm{Mo}_{0}}=0 \cdot 20 .\right.
$$

Our results have been given in figure 11 at temperatures $200 \mathrm{~K}, 400 \mathrm{~K}$ and $600 \mathrm{~K}$. Though $\mathrm{D} 1_{a}$ is the stable equilibrium structure at $c_{\mathrm{Mo}}=0.20$, a stronger tendency for the development of $\left\langle 1 \frac{1}{2} 0\right\rangle^{*}$ ordering can be noticed in the initial stages of ordering as reflected by a larger negative curvature of the free energy surface at $\eta_{n}^{\left[1 \frac{1}{2} 0\right]}=\eta_{n}^{\frac{1}{5}[420]}=0$ along the $\eta_{n}^{\left\lfloor\frac{9}{2} 0\right]}$ axis.

At $T=200 \mathrm{~K}$, the curvature of free energy surface is negative in both the directions which suggests that homogeneous ordering is possible for both the ordering processes. A mixed state consisting of concentration waves with wave vector ranging from $\left\langle 1 \frac{1}{2} 0\right\rangle^{*}$ to $\frac{1}{5}\langle 420\rangle^{*}$ is encountered on the path of the ordering process at these temperatures.

From the above analysis, it is clear that the first-principles based free energy calculations can be used for the determination of thermodynamic tendencies driving a transformation process involving several competing superstructures and rationalizing the path of a transformation when a first-order and a second-order ordering processes are competing.

\section{Summary}

The short-range and long-range ordering processes occurring in the Ni-Mo alloys have been studied theoretically and are compared with the experimental results. The stability sequence of these superstructures as observed experimentally is in agreement with the results of the first-principles calculations. Free energies of these ordered structures have been calculated on the basis of ASR-OP formalism in conjunction with the SCW model. Free energy-order parameter plots obtained from such calculations have also identified the regimes of ordering instabilities. It has been demonstrated that such an approach can be used to study the complex transformation behaviour involving several competing superstructures as well as competing first order and second order ordering processes. 


\section{Acknowledgements}

The authors would like to thank Prof. A Mookerjee, Dr T Saha-Dasgupta and Dr I Dasgupta for many helpful discussions.

\section{References}

Andersen O K, Jepsen O and Glötzel D 1985 in Highlights in condensed matter theory (eds) F Bassani, F Fumi and M P Tosi (Amsterdam: North Holland) p. 59

Andersen O K 1987 in Electronic band structure and ifs applications, Lecture notes in physics (ed.) $\mathrm{M}$ Yussouf (Berlin: Springer) vol. 283

Arya A, Das G P. Banerjee S and Patni M J 1997a Phys. Rev. B (communicated)

Arya A, Das G P, Banerjec S. Saha-Dasgupta T, Dasgupta I and Mookerjee A 1997b (to be published) Banerjee S, Kulkani U D and Urban K 1989 Acta Metall. 3735

Banerjee S, Urban K and Wilkens M 1984 Acta Metall. 32299

Brooks C R, Spruiell J E and Stansbury E E 1984 in Physical metallurgy of nickel-molybdenzm alloys, Inter. Metal Rev. 29210

Casselton R E W and Hume-Rothery W 1964 J. Less-Common Met. 7212

Das S K, Okomoto P R, Fischer P M J and Thomas G 1973 Acta Metall. 21913

Das S K and Thomas G 1974 in Order-disorder transformation in alloys (ed.) H Warlimont (Berlin: Springer) p. 332

Fontaine D de 1975 Acti Metull. 23553

Fontaine D de 1981 in Solid-state phase transformations (eds) H I Aaronson et al (Pennsylvania: Metall. SOc. A.I.M.E) p. 25

Guthrie P V and Stansbury E E 1961 in X-ray and metallographic study of the nickel-rich alloys of the Ni-Mo system, Vol, II, Report ORNL 3078, (Tenn: Oak Ridge National Laboratory)

Hansen M and Anderko K 1958 in Constitution of binciry alloys (New York: McGraw-Hill)

Heijwegen C P and Rieck G D 1973 \%. Metallkd. 64450

Kear B H, Sims C T, Stoloff N S and Westbrook J H (eds) 1970 in Ordered alloys (Baton Rouge: La Claitor's Publishing)

Khachaturyan A G 1973 Plyss. Status Solidi 980

Khachaturyan A G 1983 Theory of structural transtormations in solids (New York: Wiley)

Kulkarni U D and Banerjec S 1988 Acta Metall. 36413

Kulkarni U D, Banerjee S and Kulkarni S D 1993 Acta Metall. Mater. 411283

Martin P L and Williams J C 1984 Acta Metall. 321681

Mookerjee A 1994 in Methods of electronic structure calculations (eds) O K Andersen, V Kumar and A Mookerjee (Singapore: World Scientific) p. 193

Ridder R De, Tendeloo G Van and Amelinckx S 1976 Acta Crystallogr. A32 216

Shunk F A 1969 in Constitution of binary alloys (New York: McGraw-Hill) Second suppl.

Tendeloo G Van 1976 Mater. Sci. Engg. 26209

Tendeloo G Van, Amelinckx $S$ and Fontaine D de 1985 Acta Crystallogr. B41 281

Yamamoto M, Shohno F and Nenno S 1978 Trans. Jap. Inst. Metals 19475

Warlimont $\mathrm{H}$ (ed.) 1974 in Order-disorder transformation in allows (New York: Springer-Verlag)

Westbrook J H (ed.) 1967 in Intermetallic compounds (New York: Wiley) 